\title{
El modelo de servicio de un MBA con enfoque humanístico
}

The service model of a MBA with humanistic approach

Le modèle de service d'un $M B A$ avec une approche humaniste

Ernesto Barrera Duque

ernesto.barrera@inalde.edu.co

Autopista Norte Km. 7 ,

Costado Occidental,

INALDE, Chía,

Cundinamarca-Colombia

Ph.D. en Administración Universidad EAFIT. MBA, Master en Dirección de Empresas, IESE

Business School. Especialista

en Economía Internacional

y Abogado Universidad

Externado. Profesor de

Marketing, INALDE Business

School, Universidad de La

Sabana. Vinculado al grupo

de investigación "Marketing

Estratégico” de INALDE.

Artículo Tipo: de investigación Según Clasificación Colciencias

Fecha de recepción: 7 de Febrero de 2011 Fecha de corrección:

22 de junio de 2011

Fecha de aprobación: 22 de junio de 2011

\section{Resumen}

Este artículo de investigación tiene como objetivo establecer los elementos fundamentales de la experiencia del estudiante MBA en el programa de una institución educativa colombiana. Mediante la combinación del análisis narrativo y la teoría fundamentada, ambos dentro de la modalidad de metodología cualitativa, se hallaron categorías fundamentales en la experiencia de servicio. La interacción con los profesores, la interacción con los directores de estudio, el contexto organizacional de la institución educativa, la interacción con otros participantes y el diseño del programa centrado en el participante, fueron los inductores de la experiencia. Además, se encontró que el método del caso era un mediador importante para verter una experiencia de carácter práctico. Finalmente, los elementos de impacto en el estudiante fueron el desarrollo de habilidades directivas y la transformación personal integral. El enfoque humanístico, imbuido en todas las categorías, sirvió de pliegue entre ellas, indicando que este elemento es consistente y potencia la formación orientada a mejorar el desempeño laboral y personal de los participantes. Además, este enfoque, que es evidente en la experiencia, es valorado por los estudiantes.

Palabras clave: servicios, experiencia del cliente, MBA, enfoque humanístico, teoría fundamentada, métodos cualitativos, análisis narrativo. 


\section{The service model of a MBA with humanistic approach}

\author{
El modelo de servicio de un MBA con \\ enfoque humanístico
}

Le modèle de service d'un MBA avec une approche humaniste

\begin{abstract}
This research had the objective to establish the key elements of a student's experience in a Colombian MBA program. Through narrative analysis and grounded theory, both qualitatitive methods, key categories were found for service experience. Professor's interaction, couch's interaction, organization's context, interaction with other students, and participant centric program design, were key drivers in this experience. Besides, case method was found as an important mediator in delivering a practical experience. Finally, the consequences of the experience were: skill development and integral personal transformation. The humanistic focus embedded in each category was a connector among them, and confirming its importance for an oriented performance education. The participants appreciate this humanistic approach.
\end{abstract}

Keywords: services, customer experience, MBA, humanistic approach, grounded theory, qualitative methods, narrative analysis.

\section{Le modèle de service d'un MBA avec une approche humaniste}

\author{
El modelo de servicio de un MBA con \\ enfoque humanístico
}

The service model of a MBA with humanistic approach

\section{Résumée}

Cet article de recherche a pour objectif celui d'établir les éléments fondamentaux de l'expérience de l'étudiant de MBA dans une institution d'éducation colombienne. À partir de la combinaison de l'analyse narrative et de la théorie fondée, encadrées par la modalité de méthodologie qualitative, on a pu trouver des catégories fondamentales sur l'expérience de service. L'interaction avec les professeurs ainsi qu'avec les directeurs d'études, le contexte organisationnel de l'institution d'éducation, l'interaction avec d'autres participants et la conception du programme centrée sur le participant, ont été les inducteurs de l'expérience. En plus, on a trouvé que la méthode de cas était un médiateur important pour appliquer une expérience de type pratique. Finalement, on a identifié comme éléments d'impact sur l'étudiant le développement de capacités de direction et une transformation personnelle intégrale. L'approche humaniste, inculquée dans toutes les catégories, a servi comme pli entre celles-ci, indiquant ainsi que cet élément est consistant et favorise la formation orientée à améliorer la performance personnelle et professionnelle des participants. En plus, cette approche, qui se montre évidente sur l'expérience, est appréciée par les étudiants .

Mots clef: services, expérience du client, MBA, approche humaniste, théorie fondée, méthodes qualitatifs, analyse narrative. 


\section{El modelo de servicio de un MBA con enfoque humanístico}

\section{Introducción}

LOS programas MBA (maestrías en administración o dirección de empresas) podrían considerarse una categoría madura en el mundo desarrollado. No obstante, se halla en su fase final de crecimiento en países en vías de desarrollo, entre ellos Colombia. Ahora bien, críticas como la exacerbación de la codicia, el relajamiento de los límites éticos, el énfasis en la racionalidad técnicoinstrumental, la alienación de las mentes con el sistema capitalista sin rostro humano, la deshumanización, han aumentado en los últimos años. Sin embargo, esta investigación, se aclara, estuvo impulsada por una mirada optimista de esta categoría de servicio educativo y de su potencial para impactar en el mejoramiento del oficio de la dirección y en la transformación social.

Una mezcla de análisis narrativo con la teoría fundamentada, fue el método utilizado en esta investigación cualitativa. Esto indujo a excluir un marco teórico previo, ya que el investigador dejó que las categorías y las relaciones entre éstas emergieran de la evidencia empírica. La conceptualización fue el punto de llegada.

Este documento se ha organizado en varias secciones: La primera, corresponde a esta introducción. La segunda, describe la metodología utilizada. La tercera, se dedica a los resultados, es decir, a la síntesis interpretativa de donde emerge el modelo conceptual. La cuarta sección, por su lado, corresponde a la conclusión, donde se han incluido las limitaciones y las avenidas para investigaciones futuras.

\section{Metodología}

La pregunta general de investigación que orientó este estudio exploratorio consistió en comprender "¿cómo se genera y vive la experiencia educativa de los estudiantes en el programa MBA del INALDE ${ }^{1}$ ?" Para recopilar los datos cualitativos para este estudio, se abordaron 58 estudiantes que terminaron su primer semestre en $2010^{2}$, y se les solicitó, que de manera anónima, escribieran abiertamente durante treinta minutos acerca de su experiencia integral en el programa; orientados por la evocación: "Narre y cuente su experiencia (pensamientos, sentimientos, emociones, sensaciones, relaciones) durante este primer semestre del MBA".

Todas las narrativas escritas fueron escaneadas y se incorporaron a un archivo organizado y sistematizado (electrónico y físico). Se utilizó el software cualitativo Nvivo $\mathbf{8}$, que además, sirvió de soporte para la codificación, el análisis narrativo y la síntesis interpretativa.

La base fue la mezcla de análisis narrativo (Patton, 2002) y teoría fundamentada (Glaser y Strauss, 1967; Strauss y Corbin, 2002), utilizando para el efecto las técnicas de identificación de patrones comunes, la codificación abierta, y la triangulación de informantes (Patton, 2002). No se utilizó un marco teórico o conceptual específico previo con el cual iterar, ya que el objetivo era precisamente conceptualizar a partir de la perspectiva directa de los estudiantes ${ }^{3}$, buscando un modelo emergente y derivado de los datos cualitativos, acogiendo, en este sentido, la variante de la postura de la teoría fundamentada propuesta por Glaser y Strauss (1967), y Strauss y Corbin (2002).

\section{Resultados}

Los estudiantes narraron por escrito - de forma espontánea y abierta- aspectos de su experiencia durante el primer semestre del programa. Se extrajeron los patrones comunes de los textos, para luego traducirlos en

1. INALDE Business School, Escuela de Dirección y Negocios de la Universidad de La Sabana, Bogotá, Colombia.

2. En 2010 ingresaron 160 estudiantes nuevos al primer año del programa MBA del INALDE. La matrícula para los dos años, era, para ese año, fue de US\$37.000.

3. En este documento se utiliza las palabras estudiante y participante indistintamente. 
categorías emergentes que identificaron, desde la perspectiva de los estudiantes, los elementos de la experiencia educativa.

\subsection{Síntesis interpretativa de las "narrativas" de los participantes}

A continuación, se presenta una síntesis interpretativa organizada por categorías emergentes y en cierto orden de importancia en función de la frecuencia, y también, sus relaciones con otras categorías. Estas categorías son: la transformación personal y el desarrollo de habilidades directivas como efectos de la experiencia, y, como inductores, la interacción con los profesores, la interacción con los directores de estudio, el contexto organizacional de la institución educativa, la interacción con otros participantes (capital social), y el diseño del programa centrado en el participante.

\subsubsection{La transformación personal integral}

Los participantes afirman que atraviesan por un proceso de transformación personal integral. Esto implica que el beneficio no es sólo la absorción de conocimientos técnicos duros, sino la adquisición y desarrollo de nuevas maneras de afrontar, mirar y abordar el mundo. Recuperan y aprehenden criterios y valores para actuar coherentemente en sus familias, en sus trabajos y en la sociedad:

"He sentido un impacto impresionante en mi vida como persona y en los criterios que tengo que tener como directivo y aplicarlo en todos los aspectos de mi vida."

Obsérvese en el mismo sentido, entre otras, la siguiente aseveración

"El punto importante es que el INALDE no sólo forma profesionales sino personas y es aquí la gran fortaleza de esta institución.”

Este ejemplo de evidencia confirma que, desde la perspectiva de los estudiantes, la formación integral con impacto transformativo se percibe como un efecto de la experiencia, no sólo desde el punto de vista técnico, sino también humano, por vía del enfoque humanístico que se imprime en el desarrollo de las sesiones. Y, además de la transmisión de "saberes" (conocimientos) y del perfeccionamiento en el "hacer" (habilidades y competencias), perciben un impacto transformativo en el "ser". El programa MBA influye en sus valores, actitudes (se activa la preocupación por el otro, el respeto de la dignidad humana, empiezan a entender a la persona humana como fin en sí mismo y no como un medio, etc.), modelos mentales, miradas del mundo, actuaciones y comportamientos. Su participación en el programa, así, no sólo los transforma mejorando su desempeño laboral, sino también, influye en el ámbito de sus relaciones familiares, sociales y de ciudadano

"Es una experiencia que ha transformado positivamente mi modelo mental frente a las decisiones a las cuales me veo enfrentado en el ámbito laboral y también familiar y social."

Ahora bien, esta transformación personal integral se materializa en la interacción sucesiva con profesores y participantes, en un contexto organizacional específico, $y$, con la ayuda del método del caso. Es el resultado de la interacción humana personalizada entre profesor y estudiante, que le permite a éste apreciar, valorar, absorber, vivir y reconocer la fuerza transformativa del programa en el saber, el hacer y en el ser, desplegada, en el aula de clase, con las preguntas y contra-preguntas, las discusiones entre estudiantes, los cierres de los profesores, las retroalimentaciones personalizadas, en el trato por fuera del aula.

El conocimiento co-construido socialmente, con la facilitación del profesor, se replica, se vierte en el comportamiento práctico de los estudiantes por fuera de la institución educativa, en sus vidas laborales y familiares concretas

"Pienso que he avanzado como persona (en mi, en mi hogar, en mi trabajo, en mi familia) con lo cual el balance me dice que el esfuerzo y dedicación vale la pena y tiene sentido."

La institución educativa se erige así, para el estudiante, en un componente del punto de inflexión en la activación de su propia búsqueda de sentido o de propósito vital, una guía y orientación para ayudarle en el camino hacia los valores humanos fundamentales, hacia la construcción y descubrimiento del faro orientador y de criterios para sus acciones en medio del mundo. De modo que una proposición es 
que el programa, desde la perspectiva de los estudiantes, tiene un impacto positivo en su desempeño laboral y en su crecimiento humano integral (profesional, laboral, familiar, individual; en el saber, en el hacer, en el ser). Impulsa una transformación personal integral orientada por la institución educativa que imprime un enfoque humanístico entendido como el respeto profundo a la dignidad del ser humano, y postula que la persona humana es el centro de la organización. Y, los estudiantes reconocen e identifican el interés de la institución y de los profesores por desarrollar mejores personas para la sociedad, no sólo mejores directivos.

“Mi primera impresión positiva está relacionada con el valor que en Inalde se le da a la persona. Acá yo me he sentido especial, importante."

"Me siento más que un estudiante una persona."

Todo esto, al final, implica una transformación positiva integral del participante, no hacia la codicia o el egoísmo, sino a la preocupación del impacto de sus acciones en otros: contribución. De otro lado, obsérvese este texto

"Algo que me tranquiliza con mis desaciertos en esta nueva experiencia es que ahora soy consciente de mis defectos más que cuando comencé, soy mejor que hace seis meses, he crecido y realmente no estoy compitiendo por la nota sino conmigo mismo."

En este testimonio hay una mención especial a la introspección que el programa incentiva en los participantes: "soy consciente de mis defectos", dice. Los estudiantes activan la reflexión sobre sí mismos y sobre sus relaciones con los demás. Desarrollan el pensamiento crítico para cuestionarse a sí mismos y para reconocer, rescatar o descubrir lo valioso que hay en el Otro. Así parezca paradójico o contra-intuitivo, el programa les activa la virtud de la humildad en el ejercicio del oficio directivo, que, en este caso, en el del testimonio transcrito (como narrativa representativa), les ayuda a realizar diagnósti-

\begin{tabular}{|c|c|c|}
\hline Categoría & Dimensiones & Especificidades \\
\hline \multirow{8}{*}{ 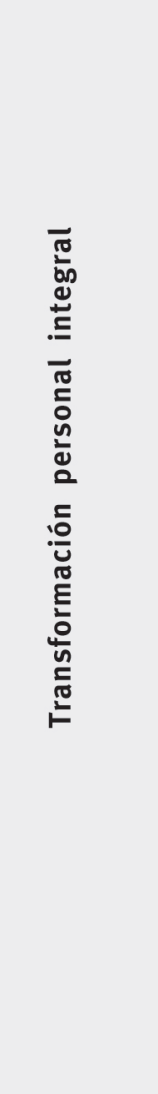 } & Crecimiento personal & ser mejor integralmente (multidimensionalidad) \\
\hline & Recuperación de valores & comportamiento uniforme en sus vidas cotidianas \\
\hline & Unidad de vida & $\begin{array}{l}\text { * familia, trabajo, individuo, ciudadano } \\
\text { * el mismo en todas las dimensiones de su vida cotidiana }\end{array}$ \\
\hline & Formación integral & $\begin{array}{l}\text { * saber, hacery ser } \\
\text { * laboral, personal, familiar, ciudadano }\end{array}$ \\
\hline & $\begin{array}{l}\text { Interacción con los } \\
\text { profesores }\end{array}$ & $\begin{array}{l}\text { * humana personalizada } \\
\text { * retroalimentación uno a uno } \\
\text { * enfoque humanístico: desarrollar y potenciar la persona } \\
\text { humana } \\
\text { * desarrollar mejores directivos, con criterio prudencial } \\
\text { * motivación contributiva del profesor (concreta y abstracta) }\end{array}$ \\
\hline & $\begin{array}{l}\text { Apoyo y activación de la } \\
\text { búsqueda de un sentido o } \\
\text { propósito vital }\end{array}$ & $\begin{array}{l}\text { * proyecto de vida: un sentido o propósito en medio del } \\
\text { mundo } \\
\text { * co-construir con los estudiantes un faro orientador para sus } \\
\text { vidas }\end{array}$ \\
\hline & Mejor desempeño laboral & percibido de manera inmediata, aplicabilidad en tiempo real \\
\hline & Introspección & $\begin{array}{l}\text { * desarrollo de la inteligencia intra-personal y moral } \\
\text { (autoconocimiento) } \\
\text { * activar su propia reflexión interior orientada al propósito } \\
\text { vital } \\
\text { * ejercicio de la virtud de la humildad }\end{array}$ \\
\hline
\end{tabular}


cos realistas sobre sí mismos y a pensar acerca del sentido o propósito en medio del mundo.

Por tanto, el programa impacta positivamente en su transformación integral, incluso, en la reconstrucción de sus actitudes y valores en las diferentes dimensiones vitales. Y esto, además, tiene un impacto para la institución educativa y para los que ejercen el oficio de la docencia en este contexto de formación de directivos con experiencia: un sentido o propósito para su trabajo como profesor. Obsérvese la frase "soy mejor que hace seis meses" que muestra, desde la perspectiva del participante, que el trabajo que hace el profesor "los mejora" en distintas dimensiones. Al desvelar esto ante el docente, éste deviene consciente del impacto positivo y transformador de su trabajo en Otros, lo que potencia su propia motivación contributiva, es decir, contribuir para que el Otro sea mejor en todas sus dimensiones, para que su desempeño integral se potencie.

"Realmente estoy sorprendido de la calidad del programa. Creo que éste no es un programa de estudios, sino un programa para la vida. Siento sinceramente que estoy creciendo como persona y como directivo."

En la página anterior la Tabla 1, despliega la categoría emergente denominada transformación personal integral.

\subsubsection{El desarrollo de habilidades directivas}

El “mejor desempeño laboral relativo" fue hallado como efecto de la experiencia o beneficio percibido por los estudiantes, pero también como una de las dimensiones de la categoría denominada transformación integral o crecimiento personal integral que, además, in vivo implica el perfeccionamiento en el "saber hacer".

Se encontró que los estudiantes perciben rápidamente un mejoramiento en su propio desempeño directivo. Sienten que van perfeccionando sus habilidades de análisis, de síntesis, de toma de decisiones, de comunicación, de dirección de personas, de argumentación, de trato con los demás, apoyados, como ellos mismos lo expresan, en la interacción permanente con los profesores, dentro del aula de clase y fuera de ella, soportados en el método del caso como estrategia pedagógica fundamental. Esta metodología, por novedosa, causa incluso frustración en algunos, aunque, rápidamente, terminan apreciándola por el impacto positivo en el perfeccionamiento práctico de sus capacidades directivas. Y además de ser un mediador en el desarrollo de habilidades directivas, el método del caso también tiene un impacto: la "gestión" de sus propias vidas cotidianas:

"El método del caso lo estoy llevando a mis sesiones de trabajo y personales para plantear los problemas de manera ordenada y orientada a solucionarlos."

A continuación se muestra la Figura 1 que vincula, como se ha derivado de los datos cualitativos, las relaciones entre la interacción con los profesores, la transformación integral, el desarrollo de habilidades directivas, y la mediación pedagógica del método del caso:

Figura 1. Transformación personal y desarrollo de habilidades directivas

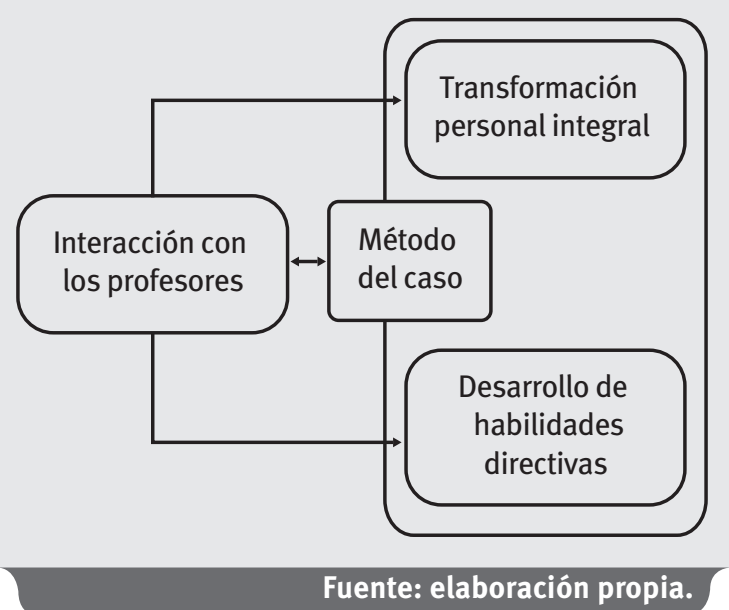

Los estudiantes también perciben que el programa MBA les desarrolla y potencia el pensamiento crítico, habilidad fundamental en la propuesta humanística y que también aparece en los lineamientos gubernamentales para las maestrías en Colombia. Esta habilidad es un elemento esencial para el despliegue directivo práctico, especialmente para los que tienen una amplia experiencia laboral, ya que les ayuda a cuestionar (crítica constructiva) sus propios supuestos, así como los que han internalizado sobre cómo se comportan las personas, las empresas y los sectores. 


\begin{tabular}{|c|c|c|}
\hline Categoría & Dimensiones & Especificidades \\
\hline \multirow{6}{*}{ 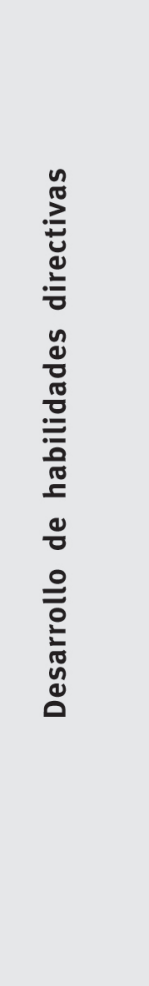 } & Saber hacer & $\begin{array}{l}\text { * mejoramiento del desempeño laboral vía habilidades } \\
\text { laborales } \\
\text { *aplicación en tiempo real de los aprendizajes }\end{array}$ \\
\hline & Pensamiento crítico & $\begin{array}{l}\text { * cuestionarse a si mismo y cuestionar la sabiduría } \\
\text { convencional (entre otras) } \\
\text { * virtud de la humildad }\end{array}$ \\
\hline & $\begin{array}{l}\text { Pensamiento integrador } \\
\text { (para la toma de decisiones) }\end{array}$ & $\begin{array}{l}\text { capacidad de sintesis de las diferentes funciones y } \\
\text { perspectivas }\end{array}$ \\
\hline & $\begin{array}{l}\text { Pensamiento creativo e } \\
\text { innovador }\end{array}$ & para soluciones y planes de acción \\
\hline & Crecimiento profesional & $\begin{array}{l}\text { * análisis } \\
\text { * sintesis } \\
\text { * criterios prudenciales para la toma de decisiones } \\
\text { comunicación y argumentación } \\
\text { * trato humanizado con el Otro (enfoque humanístico } \\
\text { aplicado al propio trabajo) } \\
\text { * desarrollo de la inteligencia interpersonal y el trabajo } \\
\text { en equipo }\end{array}$ \\
\hline & $\begin{array}{l}\text { Interacción con los } \\
\text { profesores }\end{array}$ & $\begin{array}{l}\text { * desarrollo de criterios directivos } \\
\text { * humana y personalizada } \\
\text { * método del caso }\end{array}$ \\
\hline
\end{tabular}

Fuente: elaboración propia.

"Me atrae porque para personas como yo con experiencia me ayuda a formar pensamiento crítico en el desarrollo de mis actividades en mi empresa."

Los estudiantes reconocen y perciben la importancia de los criterios directivos adquiridos por vía de la interacción con los profesores (y con sus demás compañeros de sección), que además, por tratarse de una modalidad ejecutiva (tiempo parcial), aplican de manera inmediata en su trabajo

"Al principio consideré que manejaba muchos de los temas por mi experiencia, pero oh! sorpresa, tengo muchísimas cosas que he aprendido, criterios que necesito revaluar."

Las interacciones dinámicas con los profesores, con los otros compañeros de sección, con los directores de estudio, con el método del caso, con el diseño del programa y con el contexto organizacional de la institución educativa, van impactando en la transformación integral de los estudiantes y en el desarrollo de sus habilidades directivas, en términos de análisis, síntesis, trabajo en equipo, comunicación, coordinación, integración en la toma de decisiones, argumentación, escucha, entre otras, y especialmente, en el ejercicio habitual del pensamiento crítico y la virtud de la humildad.

Arriba se muestra la Tabla 2 para la categoría emergente denominada desarrollo de habilidades directivas.

Este proceso de transformación y crecimiento personal integral (en los niveles de ciudadano, familia, trabajo, personal; así como del saber, hacer y ser), de introspección (autoconocimiento), de ejercicio habitual del pensamiento crítico, de co-construcción social del conocimiento, de desarrollo de sus habilidades directivas, de reflexión sobre el sentido o propósito para sus vidas (guiados por un faro humanístico insertado en la institución educativa), de interacciones humanas personalizadas con los profesores, con los directores de estudio (que será tratado en mayor profundidad más adelante), con los otros compañeros, mediadas por el método del caso, impactan en el desarrollo de sus habilidades directivas, en la adquisición de criterios prudenciales de análisis, de decisión, de ejecución y también en una apertura a la virtud de la humildad. 


\begin{tabular}{|c|c|c|}
\hline Categoría & Dimensiones & Especificidades \\
\hline \multirow{6}{*}{ 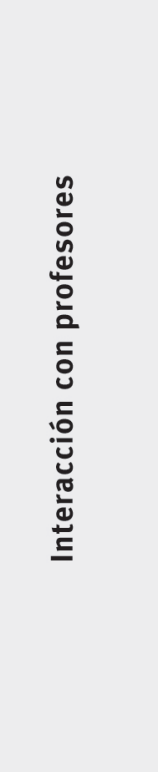 } & Personalizada & $\begin{array}{l}\text { * retroalimentación } \\
\text { * preguntas, contra-preguntas, preguntas de profundización }\end{array}$ \\
\hline & Estilo humano & $\begin{array}{l}\text { * condiciones humanas del profesor } \\
\text { * ejemplo para sus estudiantes: ejercicio de la virtud de la } \\
\text { humildad en el profesor } \\
\text { * empatía } \\
\text { * escucha activa } \\
\text { * familiaridad/cercanía } \\
\text { * acceso/disponibilidad/ejemplo de virtudes directivas }\end{array}$ \\
\hline & $\begin{array}{l}\text { Orientada al mejoramiento } \\
\text { integral del participante }\end{array}$ & $\begin{array}{l}\text { * vínculo solidario y de doble vía (flujo dialógico) } \\
\text { * co-construcción y co-aplicación del conocimiento }\end{array}$ \\
\hline & $\begin{array}{l}\text { Motivación contributiva } \\
\text { del profesor }\end{array}$ & formar y mejorar a Otros como personas y directivos (integral) \\
\hline & Excelencia académica & alta preparación intelectual \\
\hline & Experiencia práctica & contacto directo con la vida directiva y empresarial \\
\hline
\end{tabular}

Fuente: elaboración propia.

\subsubsection{La interacción con los profesores}

Esta categoría emergente se operacionali$z a$, entre otras, con la dimensión "personalizada" (interacción humana personalizada con los profesores), que se despliega por vía de preguntas en clase, preguntas de profundización, enseñanzas durante las discusiones, las retroalimentaciones, y, es, además, una de las causas de las dos categorías emergentes ya analizadas: la transformación personal integral y el desarrollo de habilidades directivas (Figura 1).

Los estudiantes valoran y reconocen, en narrativas coincidentes, la excelencia académica y las condiciones humanas de los profesores. Obsérvese, como representativo, el siguiente texto.

"La experiencia ha sido muy positiva, me impresiona el excelente nivel de los profesores y sus condiciones humanas."

Los estudiantes perciben y sienten que los profesores están realmente comprometidos con su mejoramiento como personas y como directivos. Esto es consistente con sentido central del oficio del profesor en el contexto organizacional de la institución educativa: la formación integral y humanística, el mejoramiento de sus "estudiantes", la contribución para que el otro sea mejor persona en todas sus dimensiones, y para que aporte a la trans. formación social desde su oficio directivo. El trabajo del profesor no consiste en verter la racionalidad instrumental egoísta en los cerebros y corazones de los estudiantes, para quizá aumentar su propio prestigio o cumplir con una misión de adoctrinamiento dentro de un sistema capitalista sin rostro humano. Por el contrario, en esta institución educativa, el profesor construye un vínculo solidario con el Otro, con el estudiante, para mejorarlo como ser humano integral, no sólo en su desempeño laboral. Y así lo percibe el estudiante.

El profesor irradia ante los participantes la imagen de que se ve así mismo en un flujo dialógico de crecimiento bidireccional, como co-constructor del proceso social de generación y aplicación del conocimiento, y del desarrollo de habilidades directivas. Obsérvese los siguientes testimonios representativos

"Los docentes dan lo mejor de sí para que nosotros aportemos más."

"La experiencia ha sido de crecimiento personal en cuanto a que cuando un profesor te contrapregunta o te coloca en una posición contraria, la cual no ha- 
bías analizado, es cuando más aprende uno a generar habilidad de plantear alternativas claras que cumplan los criterios que se plantearon. Cuando en mis participaciones me pasa esto, o el profesor lo lleva a uno en algún tema a puntualizar, así no haya sido lo que tenía preparado para la sesión, es cuando más he aprendido y quedo muy satisfecha."

Y, para finalizar esta sección, obsérvese el siguiente texto, que denota la importancia de la formación por vía del ejemplo de los profesores. Como el viejo adagio "no puedes dar lo que no tienes"; si el profesor quiere enseñar la virtud de la humildad en el oficio de la dirección, él mismo tiene que ser humilde (tabla 3).

"Se respira humildad en el equipo INALDE (administrativo y docente)."

\subsubsection{La interacción con el director de estudios}

Los participantes valoran el acompañamiento de esta "figura educativa" (que funge como coach y es una persona egresada del programa y diferente de los profesores) en su proceso hacia la formación personalizada. Materializa, para ellos, elementos como el trato humano y familiar, uno a uno, que "los hace sentir importantes". Observan en esta figura una preocupación "de verdad" de la institución por cada uno de ellos en concreto, como personas, que opera un esfuerzo por su desarrollo humano integral. Obsérvese el siguiente testimonio representativo sobre los directores de estudio.

\begin{abstract}
"Me gustaría hacer un reconocimiento especial para XXXXXX que siempre fue una persona especial con el grupo y que sentí genuinamente se preocupaba por nosotros como seres humanos, tengo una bonita experiencia de la relación con él."
\end{abstract}

El director de estudios, que está presente durante el trabajo en equipo que se realiza antes de la discusión de los casos en sesión plenaria, se erige en un apoyo integral para el estudiante, especialmente en el proceso de inserción inicial al programa, que, por su rigurosidad, exigencia y metodología, genera sentimientos positivos, pero también de frustración, por la altura del reto intelectual y de vida, requiriendo de ayudas adicionales de la institución educativa para evitar la deserción.

"Dejo de lado a los directores de grupo, quienes ayudaron a que no desistiera en los primeros meses debido al impacto que generó en mí la metodología del caso."

Los directores de estudio, encargados de los equipos de trabajo, son un impulsor importante para los participantes en su proceso de superación de las frustraciones, dudas, reflexiones negativas, ajuste con la metodología, desajustes familiares, sensación de incompetencia, etc. Son un apoyo para responder a preguntas que ellos mismos se hacen acerca de su incorporación al programa y, como se ha mencionado, para evitar la deserción, sobre todo, cuando ellos se formulan cuestionamientos personales como el siguiente.

"La pregunta es: ¿De qué vale ser un gran profesional si después de dos años está solo?”

De esta forma, la interacción personalizada con el director de estudios ${ }^{4}$ genera valores agregados para los estudiantes en la medida que los ayudan a realizar una transición adecuada hacia el método del caso, los atienden de manera personal, uno a uno en aspectos relacionados con su vida personal, familiar y laboral, y al "llevarlos de la mano" mitigan los impulsos de deserción.

En la siguiente página se presenta la Tabla 4 donde se operacionaliza esta categoría esencial en la experiencia de servicio, analizada desde la perspectiva del participante.

En la siguiente página se muestra la Figura 2 que incorpora a la Figura 1 la interacción con el director de estudios como una categoría fundamental en el modelo de la experiencia del participante, con impacto en la interacción con los profesores, con el método del caso y con los beneficios finales para el estudiante. 
Tabla 4. La interacción humana con el director de estudios

\begin{tabular}{|c|c|c|}
\hline Categoría & Dimensiones & Especificidades \\
\hline \multirow{5}{*}{ 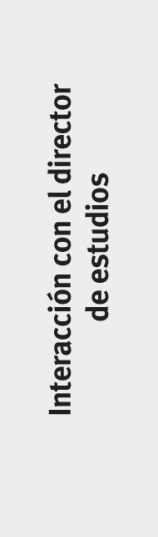 } & $\begin{array}{l}\text { Atención y asesoría } \\
\text { personalizada }\end{array}$ & los hace sentir importantes \\
\hline & Trato humano & $\begin{array}{l}\text { refleja en concreto una preocupación por ellos como } \\
\text { personas integrales }\end{array}$ \\
\hline & $\begin{array}{l}\text { Inserción inicial al } \\
\text { programa MBA }\end{array}$ & cambio de rutinas que generan frustración \\
\hline & Crecimiento profesional & $\begin{array}{l}\text { * inserción a la metodología } \\
\text { * gestión de sentimientos encontrados } \\
\text { * atiende y asesora en problemas personales } \\
\text { * aconseja cómo conciliar familia, trabajo y MBA }\end{array}$ \\
\hline & Evita la deserción & $\begin{array}{l}\text { * puente entre los profesores y directivos con el participante } \\
\text { * retroalimentación personalizada y apoyo al estilo } \\
\text { de un coach }\end{array}$ \\
\hline
\end{tabular}

Fuente: elaboración propia.

\section{Figura 2. Integración de la interacción humana con el director de estudios}

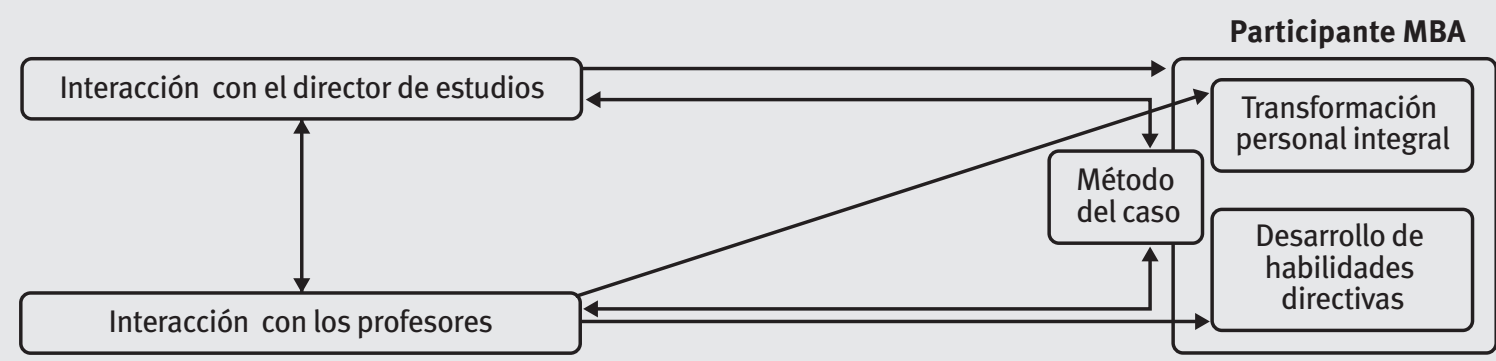

Fuente: elaboración propia.

\subsubsection{El contexto organizacional de la institución educativa}

Los estudiantes valoran de la experiencia el contexto organizacional específico de la institución educativa, es decir, en este caso, entre otras cosas, el cuidado de los detalles, la puntualidad, el aseo, los canales de comunicación, el apoyo logístico, la impresión de los casos, la infraestructura, cumplimiento en las programaciones, etc.

"Todo se tiene programado de una manera estricta."

El participante aprecia la infraestructura, la estructura, la cultura centrada en el participante, en los detalles, los matices, y los sistemas de apoyo coordinados para lograr los efectos del programa MBA. De esto deriva el profesionalismo que refleja la institución educativa.

"Se les nota el cuidado y atención que le colocan a todas las cosas."
Y manifestaciones de este estilo se encuentran de manera frecuente en sus "narrativas", obsérvense, entre otras, las siguientes.

"El programa cuenta con personas de muy alta calidad en todos los niveles, desde la dirección hasta quienes nos atienden con el café."

"Lo que más destacaría del programa es el esmero excesivo por hacer las cosas con excelencia, cuidando cada uno de los detalles y los temas de fondo."

El trato personalizado y humano como un elemento diferenciador para esta institución educativa, que no sólo se observa en los profesores, en los directivos o directores de estudio, también se hace realidad en todo el personal, se distribuye sistemáticamente por toda la institución, en todos los puntos de contacto con el estudiante, reflejando el sentido humano de la "cultura" de los detalles.

"Siempre serviciales buscan nuestra comodidad. Todas las interacciones con personas del Inalde son especiales e importantes 
todos los días y esto es lo que más me une al Inalde."

El valor percibido y la experiencia del participante están demarcados por un contexto organizacional centrado en el participante, que, según las propias narrativas de los estudiantes, se materializa en el cuidado de los detalles, la organización de todas las cosas, la puntualidad, el acceso a los directivos y profesores, la retroalimentación, el trato personalizado desde el director hasta los meseros, la preparación minuciosa de "las cosas", la preocupación por su mejoramiento integral, el ambiente de respeto y amabilidad, el orden.

Existe, además, un reconocimiento expreso, como se mencionó, a la infraestructura e instalaciones de la institución, como propicias y diseñadas particularmente para el despliegue del método del caso y para el aprendizaje en medio de la naturaleza, la comodidad y el confort.

“El área donde está ubicada la universidad es amplia, tranquila y propicia para desarrollar su objetivo."

El beneficio principal para la experiencia de servicio del estudiante, desde el punto de vista del contexto organizacional, consiste en ofrecer un ambiente de aprendizaje y crecimiento personal y profesional, de reflexión permanente sobre sí mismo y de su relación con los demás, y, adicionalmente, un espacio para co-construir y compartir sus perspectivas sobre los problemas prácticos del oficio de la dirección y confrontar con pares su experiencia directiva y de negocios. Y, además, un entorno, que por vía de la cultura, propicia y configura la personalización de la interacción de los estudiantes con los profesores y con los directores de estudio. En la página siguiente se presenta la Tabla 5 que operacionaliza el contexto organizacional centrado en el participante.

En la página siguiente se introduce la Figura 3, que representa el modelo de la experiencia de servicio (interpretada desde su propia perspectiva), donde se integra la categoría denominada contexto organizacional de la institución educativa.

\subsubsection{La interacción con otros participantes (capital social)}

La interacción con los otros participantes - lo que podría denominarse capital social de relaciones personales y profesionales de alto nivel, es considerado por los estudiantes como un elemento esencial en su proceso de formación directiva. Y no sólo implica el aspecto profesional, compartir y absorber experiencias y contribuciones de otros pares, sino también el apoyo en momentos difíciles y complejos que se presentan durante el programa. En "asociación" con el director de estudios, los participantes intervienen para evitar la deserción de sus compañeros de equipo y de sección.

También se despliega el sentido contributivo del participante (ya se había identificado en el profesor), es decir, éste no sólo busca absorber saberes y prácticas para sí mismo, también desea aportar y compartir sus conocimientos y experiencias "para que otros, con mi ayuda, sean mejores directivos".

Es un proceso de co-creación de la experiencia educativa, de co-creación del conocimiento y codesarrollo de las habilidades directivas por medio de la interacción con profesores, directivos, directores de estudio y otros estudiantes. Esto genera una “responsabilidad" para con el Otro: "Debo aportary contribuir para que el que está a mi lado sea mejor directivo y mejor persona", es decir, la motivación contributiva opera en los estudiantes.

"Pienso en todo lo que tengo por transferir de conocimiento con mi ejemplo y con mis intervenciones."

Ahora bien, se da un hecho que, aunque relevante, no mitiga este sentido contributivo del estudiante. Es cierto que existe una "competencia" al interior de las sesiones derivada de un sistema de evaluación donde la calificación depende en su mayor parte de la participación (calidad y frecuencia) oral en las sesiones, que determina si "es $\mathrm{C}$, B o A". El impulso de no estar en el $10 \%$ inferior ("C") o de posicionarse en el $10 \%$ superior ("A"), es claro que no derrumba la solidaridad en las interacciones entre ellos. Esto se observa no sólo en el apoyo emocional para evitar la deserción, sino también en las dinámicas internas durante el trabajo en equipo con el director de estudios, las sesiones plenarias, en el esfuerzo conjunto para que todos avancen en paralelo. El estudiante otorga su apoyo para que otros mejoren su desempeño académico: es una fusión de competencia y solidaridad, donde se generan vínculos solidarios y de apoyo con los "competidores", en este caso, con el compañero que está al lado, es una paradoja de ambivalencia fusionada. Se configura una interacción competitiva, pero con beneficios de doble vía: contributiva y receptiva, 
Tabla 5. Contexto organizacional centrado en el participante

\begin{tabular}{|c|c|c|}
\hline Categoría & Dimensiones & Especificidades \\
\hline \multirow{4}{*}{ 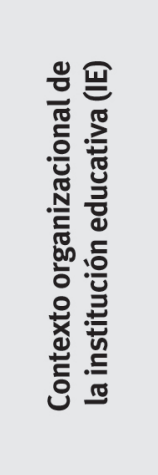 } & $\begin{array}{l}\text { Centrado en el } \\
\text { participante (ambiente de } \\
\text { aprendizaje compartido y } \\
\text { co-construido) }\end{array}$ & su mejoramiento como persona y directivo \\
\hline & $\begin{array}{l}\text { Cultura institucional } \\
\text { centrada en el participante }\end{array}$ & $\begin{array}{l}\text { * trato personalizado } \\
\text { * respeto } \\
\text { * amabilidad } \\
\text { * interés por el Otro }\end{array}$ \\
\hline & $\begin{array}{l}\text { Profesionalismo } \\
\text { institucional }\end{array}$ & $\begin{array}{l}\text { * cuidado de los detalles } \\
\text { * puntualidad y cumplimiento de los eventos }\end{array}$ \\
\hline & $\begin{array}{l}\text { Infraestructura } \\
\text { institucional }\end{array}$ & $\begin{array}{l}\text { * instalaciones cómodas en medio de la naturaleza } \\
\text { * propicia para el intercambio académico }\end{array}$ \\
\hline
\end{tabular}

Fuente: elaboración propia.

\section{Figura 3. Integración del contexto organizacional de la IE en la experiencia de servicio}

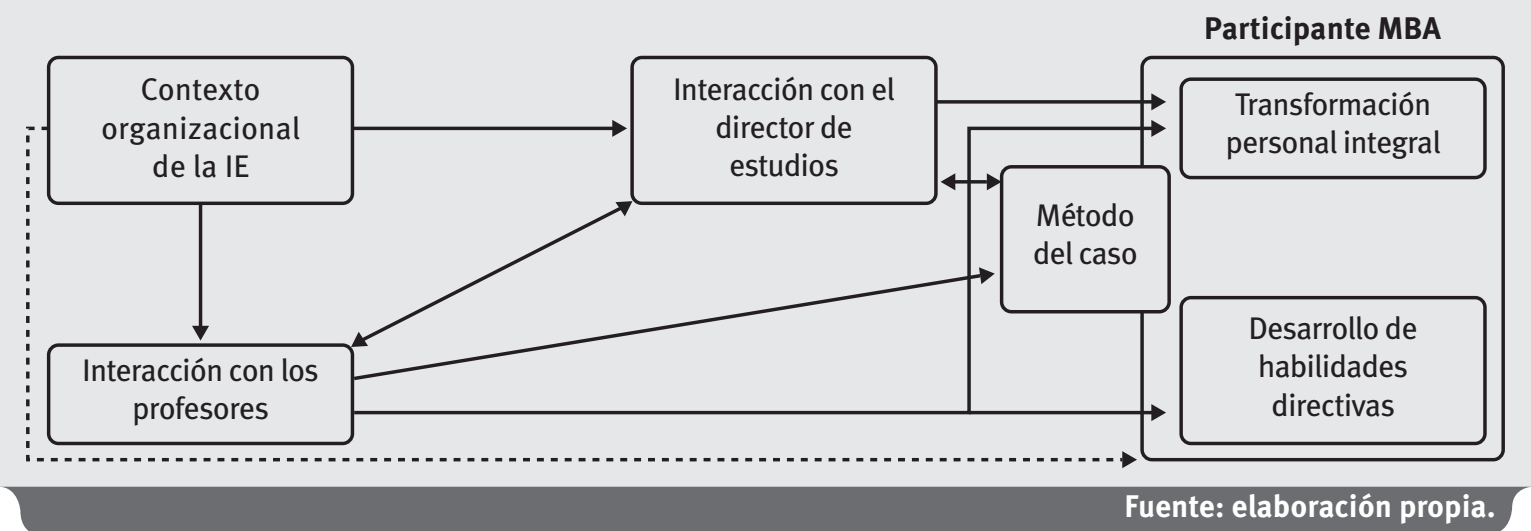

es una tensión en reciprocidad, un flujo dialógico con el Otro que despliega un proceso de codescubrimiento, de co-construcción de la experiencia académica y de co-desarrollo del proceso de perfeccionamiento directivo. $Y$ la percepción sobre el nivel de sus compañeros, los que comparten el espacio de formación, es esencial para la co-construcción de esta experiencia y para la percepción de calidad del programa.

"Adicionalmente, excelentes compañeros. He aprendido mucho de todos. Muy buena selección.”

"Representa para mí un reto y esfuerzo adicional para procurar el nivel del grupo."

El diseño del programa, como se verá en la siguiente sección, con la herramienta del método del caso como estrategia pedagógica central, implica para los estudiantes participar activamente (método centrado en el participante) dentro de un proceso de co-descubrimiento de conocimientos orientados a la ejecución, es decir, al saber hacer.

En la página siguiente se muestra la Figura 4, que integra en el modelo de experiencia de servicio la interacción con otros participantes.

En la página siguiente se presenta la Tabla 6 que operacionaliza (dimensiones y especificidades) la interacción humana con otros participantes como categoría emergente en el modelo de la experiencia de servicio del estudiante del MBA.

\subsubsection{El diseño del programa centrado en el participante}

Los estudiantes mencionan expresamente en sus narrativas que el diseño del programa es fundamental en su experiencia académica. En esta categoría emergente hay varios elementos de análisis que se presentan con cierto orden a continuación y cuyos contenidos permiten establecer que el diseño del programa ha sido consistente con la misión de la institución educa- 


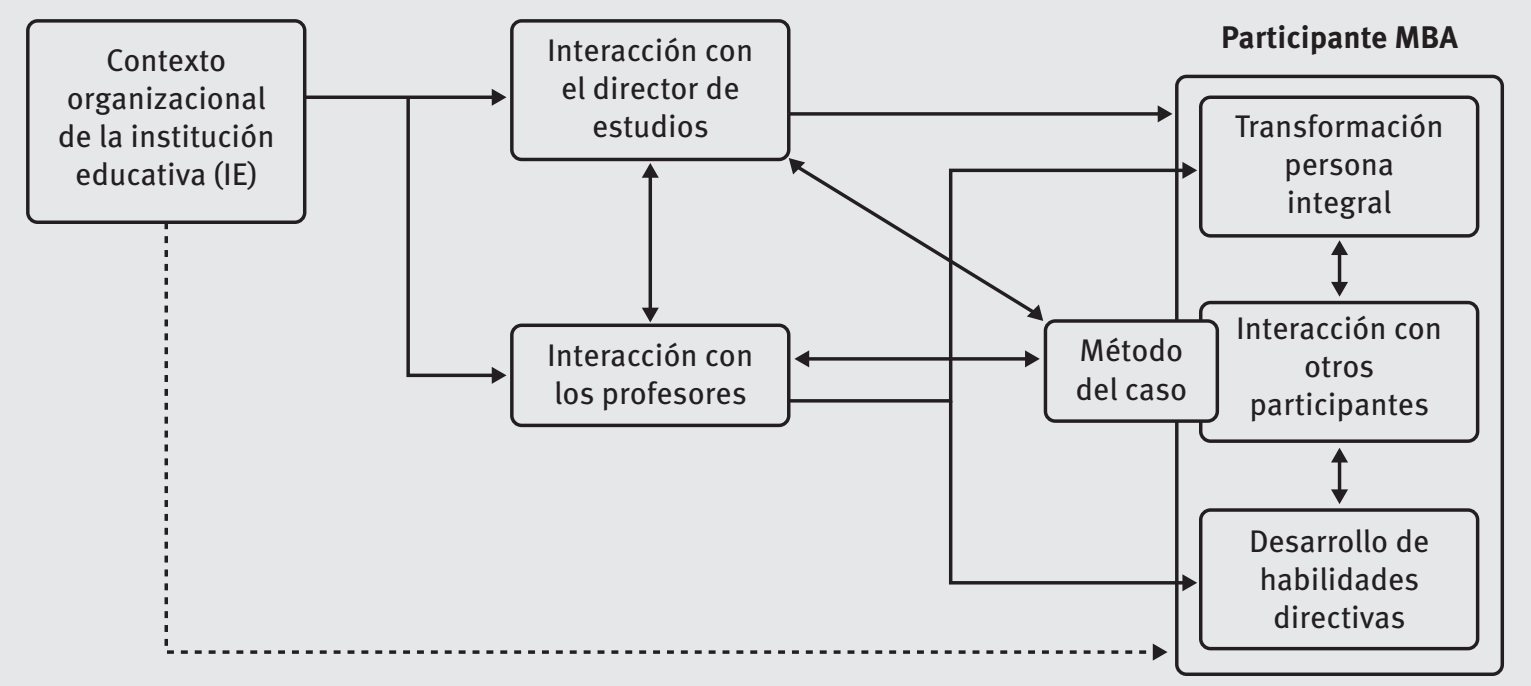

Fuente: elaboración propia.

Tabla No. 6. Interacción humana con otros participantes

\begin{tabular}{|c|c|c|}
\hline Categoría & Dimensiones & Especificidades \\
\hline \multirow{6}{*}{ 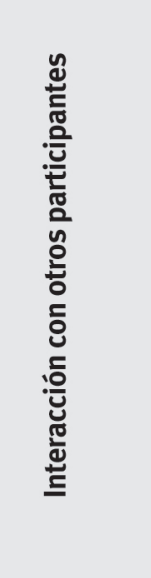 } & Capital social & red de relaciones de alto nivel \\
\hline & $\begin{array}{l}\text { Compañeros con } \\
\text { experiencia directiva }\end{array}$ & $\begin{array}{l}\text { * buena selección } \\
\text { * heterogeneidad }\end{array}$ \\
\hline & Flujo recíproco & $\begin{array}{l}\text { * co-descubrimiento y co-construcción social del } \\
\text { conocimiento y habilidades } \\
\text { * aportaciones y absorciones } \\
\text { * el método del caso como herramienta mediadora }\end{array}$ \\
\hline & Apoyo emocional & evita la deserción \\
\hline & $\begin{array}{l}\text { Contexto organizacional y } \\
\text { diseño del programa }\end{array}$ & $\begin{array}{l}\text { potencian la interacción entre participantes, y de estos } \\
\text { con los profesores }\end{array}$ \\
\hline & Motivación contributiva & $\begin{array}{l}\text { * contribución al mejoramiento de los otros participantes } \\
\text { * solidaridad contributiva en medio de la competencia } \\
\text { mutua: ambivalencia fusionada. }\end{array}$ \\
\hline
\end{tabular}

Fuente: elaboración propia.

tiva, así como con los beneficios finales para los estudiantes dentro del modelo de experiencia que aquí se ha venido elaborando: la transformación personal integral y el desarrollo de las habilidades directivas.

Los estudiantes aprecian, como ya se ha mencionado, la orientación práctica o el énfasis en el saber hacer, que permite la aplicabilidad inmediata de los conocimientos y de las habilidades adquiridas y potenciadas durante el programa, es decir, valoran la modalidad ejecutiva al permitirles iterar y confrontar con la realidad práctica lo que van aprehendiendo durante las sesiones de trabajo en equipo y en las sesiones de clase con los profesores: perciben resultados inmediatos en su desempeño laboral.
"Es muy importante poder aplicar rápidamente lo que se aprende: esto le da más sentido al programa y a su beneficio."

Y esto no sólo se refleja en su propio trabajo directivo, también, como ya se ha mencionado, en otras dimensiones de su vida de relación.

“Tanto en la familia como en la empresa he podido aplicar algunos de los aprendizajes especialmente los de 'dirección de personas'."

Valoran, igualmente, como se observa en diferentes "narrativas", el hecho de "aprender de una forma diferente" y por vía de un programa con una orientación humanística y ética. 
"El método y los contenidos de las materias es muy pertinente, así como la orientación ética."

La orientación ética del programa no se materializa sólo incluyendo dentro de su pensum una asignatura denominada "ética" como se hace tradicionalmente en las escuelas de negocios y facultades de Administración, sino que se inserta dentro del corazón mismo de todas y cada una de las materias con una visión transversal, integrando el criterio ético en el análisis de las alternativas y los procesos de toma de decisiones prácticas en los casos de todas las asignaturas: se vive en cada caso, en cada sesión de clase, en cada decisión.

De otro lado, los estudiantes mencionan de manera frecuente el cambio de rutinas inmediatamente inician el programa, lo que evidencia nuevamente la importancia del director de estudios en el proceso de inserción. Obsérvese, por ejemplo, el siguiente testimonio representativo.

\begin{tabular}{|c|c|c|}
\hline Categoría & Dimensiones & Especificidades \\
\hline \multirow{6}{*}{ 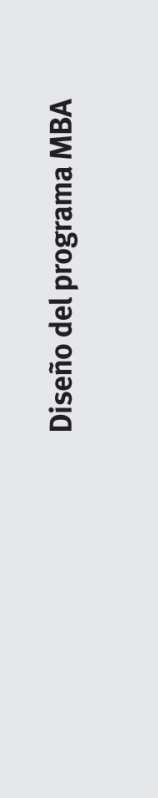 } & $\begin{array}{l}\text { Centrado en el } \\
\text { participante }\end{array}$ & $\begin{array}{l}\text { para mejorarlo como persona y como directivo (desarrollo } \\
\text { humano integral) }\end{array}$ \\
\hline & Método del caso & $\begin{array}{l}\text { * resolución de problemas no estructurados, no operativos } \\
\text { * lidiar con la incertidumbre y la ambigüedad } \\
\text { * entrenamiento en la realidad cotidiana del directivo } \\
\text { * co-descubrir y co-construir el conocimiento aplicado } \\
\text { * novedoso para ellos } \\
\text { * un halo de misterio: resolver enigmas... } \\
\text { el misterio de cada caso }\end{array}$ \\
\hline & Modalidad ejecutiva & $\begin{array}{l}\text { * orientación práctica (saber hacer) } \\
\text { * aplicación inmediata de los conocimientos } \\
\text { * iteración de los aprendizajes con la realidad práctica }\end{array}$ \\
\hline & Formación ética & como criterio transversal en todas las asignaturas \\
\hline & Rigurosidad & $\begin{array}{l}\text { * exigente } \\
\text { * esfuerzo y dedicación } \\
\text { * fuerte impacto en sus rutinas de vida } \\
\text { * reto de vida para contribuir, prepararse y lograr objetivos }\end{array}$ \\
\hline & Evaluación cualitativa & $\begin{array}{l}\text { * } A, B \text { y C } \\
{ }^{*} 10 \% \text { superior }(A) \text { vs. } 10 \% \text { inferior }(C) \\
\text { * competencia en un contexto de solidaridad y apoyo mutuo }\end{array}$ \\
\hline
\end{tabular}

Fuente: elaboración propia.

\section{Figura 5. El modelo de la experiencia de servicio del estudiante MBA}

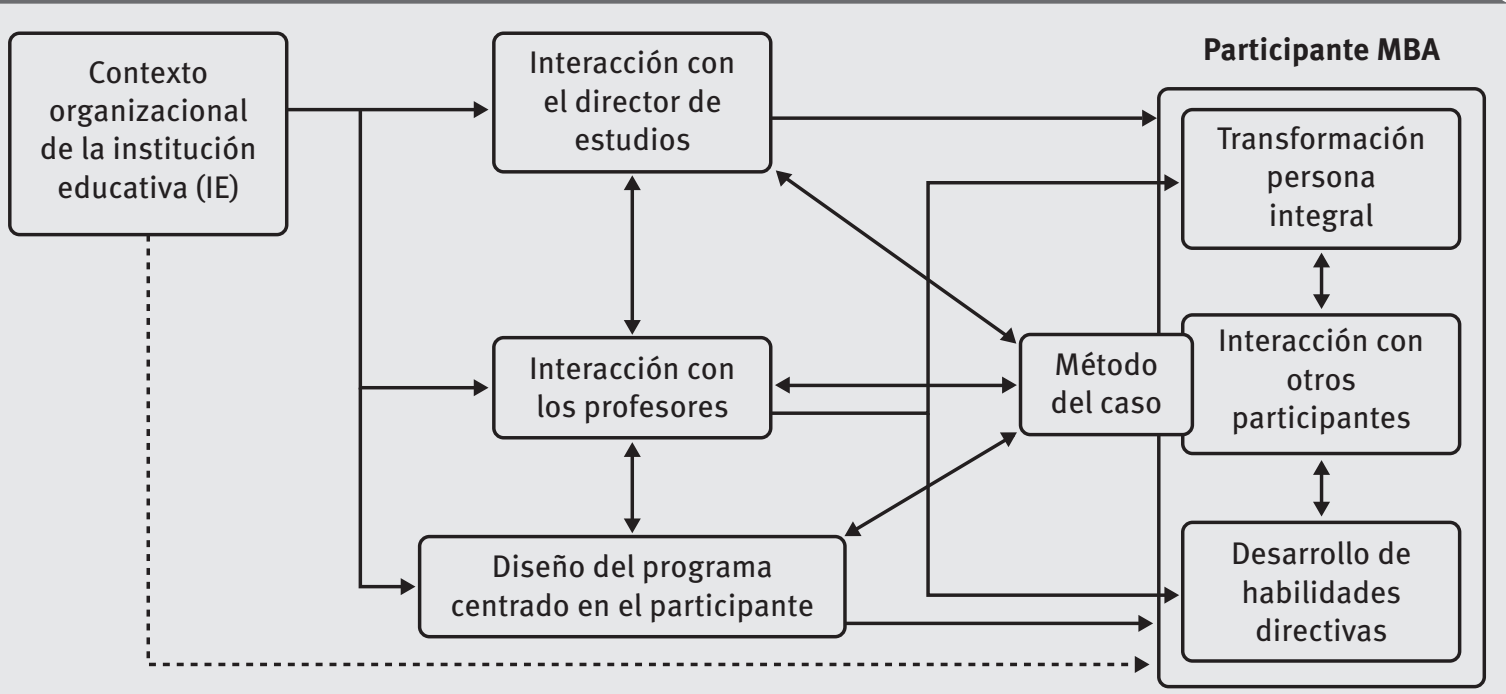


"No ha sido fácil. Iniciar este nuevo ciclo de vida como estudiante, desde el punto de vista de tiempo ha afectado algunas actividades que normalmente realizaba como montar en bicicleta y jugar tenis; esta situación no me gusta porque siento que me afecta la salud y bienestar. Este tema junto con disminuir el tiempo para mi familia y los amigos (con los cuales jugaba tenis y montaba en bicicleta) me han obligado a preguntarme si realmente esto es lo que deseaba." (registro 31).

Una de las bondades del método del caso, entendido en esta experiencia educativa como un mediador de la interacción humana personalizada (con profesores, directores de estudio y con otros participantes), es que "enseña" a los participantes "cómo pensar" con el objetivo de que sean ellos mismos quienes resuelvan los problemas que enfrentan en sus vidas cotidianas y no a través de "recetas" transmitidas por el profesor. El docente deja de ser un transmisor del "qué pensar" para tornarse en un co-constructor del "cómo pensar".

Y, además, como facilitador de "cómo" lidiar con la incertidumbre y la ambigüedad, es un apoyo para "entrenarlos" en la resolución de problemas no operativos o no estructurados, aquellos que son propios de la realidad cotidiana del directivo. Cataliza el proceso de mejoramiento en el saber hacer integral $y$, de este modo, los estudiantes empiezan a afrontar retos, no solo intelectuales 0 profesionales, sino también de sus vidas, en otras dimensiones (crecimiento personal integral).

"Creo que el programa está bien diseñado y es exigente, tratando de tener en cuenta el equilibrio entre la vida personal, profesional y como estudiante."

"En general el MBA ha sido un reto académico, intelectual y de disciplina."

En la página anterior se presenta la Tabla 7 que operacionaliza el diseño del programa centrado en el participante como categoría emergente dentro del modelo de experiencia del estudiante.

En la página anterior se presenta la Figura 5 donde se integra el diseño del programa centrado en el participante y que representa de manera completa el modelo emergente de la experiencia de servicio del estudiante.

\section{Conclusión}

El análisis, la derivación de categorías, la operacionalización dimensional, las relaciones entre categorías, representados en el modelo de la experiencia de servicio (Figura 5), explicitan una interpretación de la experiencia educativa desde la perspectiva de los estudiantes (derivada de los datos cualitativos: textos escritos con narrativas). La Figura $\mathbf{5}$, también concluye con la representación del modelo emergente hallado en esta investigación cualitativa, contiene las diferentes categorías y representa la relación entre ellas en la experiencia de servicio, con una aproximación desde la perspectiva de los estudiantes, que tienen como beneficios finales la transformación personal integral y el desarrollo de sus habilidades directivas prácticas. Los inductores encontrados de la experiencia, enlazados por el enfoque humanístico, son la interacción humana personalizada con los profesores, la interacción humana personalizada con los directores de estudio, el diseño organizacional de la institución educativa centrada en el participante, la interacción con otros participantes y el diseño del programa.

Ahora bien, el enfoque humanístico puede penetrar el funcionalismo existente en la formación de estudiantes de MBA, siempre y cuando detrás encontremos una institución educativa que, como elemento esencial de su cultura, tenga una orientación hacia desarrollo integral de sus estudiantes y que centre todos sus esfuerzos de diseño e implementación, en ese objetivo. Por tanto, aunque es importante, no es el programa MBA por sí mismo, sino el diseño organizacional de la institución educativa y la configuración de interacciones con los estudiantes, lo que más impacta en la formación de directivos con rostro humano.

Finalmente, es importante poner de relieve algunas limitaciones de este estudio: fue exploratorio, se realizó en una sola cohorte terminando su primer semestre de estudios en la modalidad ejecutiva, y fue realizado en una institución educativa que utiliza el método del caso como estrategia pedagógica central, lo que no permite una generalización externa (Maxwell, 1996), pero que se erige valiosa en cuanto que exalta las particularidades de un programa con enfoque humanístico.

Como avenidas para futuras investigaciones, la metodología de análisis narrativo podría utilizarse en otros programas MBA del país y de la región latinoamericana y establecer qué modelo de servicio emerge de los textos de los estudiantes. Esto daría espacio para encontrar semejanzas y diferencias, entendiendo que los programas tienen sus espe- 
cificidades en virtud de cada institución, y que el diseño organizacional condiciona el despliegue de la experiencia de servicio.

\section{Referencias}

Glaser, B.G y Strauss, A.L (1967). The discovery of grounded theory: strategies for qualitative research. Chicago: Aldine.

Maxwell, J. (1996). Qualitative research design. An interactive approach. Thousand Oaks: Sage.

Patton, M.Q. (2002). Qualitative research \& evaluation methods. Thousand Oaks, CA: Sage.

Strauss, A. y Corbin, J. (2002). Bases de la investigación cualitativa: Técnicas y procedimientos para desarrollar la teoría fundamentada. Medellín: Editorial Universidad de Antioquia. 